\title{
EMDR, Addictions, and the Stages of Change: A Road Map for Intervention
}

\author{
John M. O’Brien \\ Independent Practice, Portland, Maine \\ Nancy J. Abel \\ Independent Practice, South Portland, Maine
}

\begin{abstract}
A growing body of literature indicates that eye movement desensitization and reprocessing (EMDR) can be useful in the treatment of addictions. When combined with traditional addictions treatment approaches, EMDR can enhance client stability, prevent relapse, and promote recovery. Clinical decision making about when and how to use EMDR techniques with clients who present with addictions is complicated. The purpose of this article is to explore the use of EMDR interventions with clients presenting various levels of awareness of their addiction as well as varied levels of motivation to change. The authors explore the Stages of Change and suggest appropriate pre-EMDR EMDR interventions at each stage.
\end{abstract}

Keywords: addiction; EMDR; trauma; stages of change

E ye movement desensitization and reprocessing (EMDR) is an integrative psychotherapeutic approach that has been well established as an efficacious evidence-based treatment for posttraumatic stress disorder (PTSD; American Psychiatric Association $[\mathrm{APA}], 2004$; Foa, Keane, Friedman, \& Cohen, 2009; Ironson, Freund, Strauss, \& Williams, 2002; Lee, Taylor, \& Drummond, 2006). Expertise in dealing with trauma and PTSD is essential for clinicians working in the addictions field, as clients presenting with addictions often have a concomitant history of trauma with some samples indicating comorbid rates of up to $75 \%$ (Jacobsen, Southwick, \& Kosten, 2001).

Less well established in literature is the efficacy of EMDR as a treatment for addictions. The research literature is relatively sparse in documenting the uses of EMDR for clients with drug and alcohol addictions. There is even less information available to document the use of EMDR with process or behavioral addictions (e.g., gambling, sex, shopping). There is disagreement about whether or not EMDR is an effective treatment when a client is actively using substances (Abel \& O’Brien, 2010; Hase, 2010; Marich, 2009; Shapiro, Vogelmann-Sine, \& Sine, 1994).

Clients present with differing levels of awareness of addiction and different levels of motivation to change addiction-related behavior. Given such individual differences in client needs and motivation levels, effective treatment for addictions requires clinicians to individualize treatment approaches.

The purpose of this article is to explore the various ways to intervene with clients who present with addictions, including how client awareness and motivation affect treatment. After a review of extant literature, the authors will discuss the stages of change (Prochaska $\&$ DiClemente, 1992) and a model outlining various interventions tailored to the client's motivation to change. The authors then address factors to consider in implementing this model, including if and when to use the standard protocol with clients in addictions treatment.

\section{Scope of the Problem}

Many mental health clinicians resist working with clients with addictions or fail to assess these issues in the clients (Najavits et al., 1995). However, approximately $50 \%$ of clients who present to a mental health clinician have problems that are directly related to their own alcoholism or that of a family member (Drake $\&$ Mueser, 1996). The number of clients who are affected by other unaddressed addictions (e.g., sex, shopping, gambling) is not well documented 
because these disorders are frequently not diagnosed (Tamam, Zengin, Karakus, \& Ozturk, 2008). Current statistics may underestimate the true prevalence rates of addiction in the American population, and clinicians may do the same in their practices (Buchman \& Reiner, 2009; Dittmar, 2005; Del Giudice \& Kutinsky, 2007; Hollander \& Allen, 2006; van Wormer \& Davis, 2008). Mental health clinicians need to be mindful of treating addictions, whether or not they are obvious.

The costs of chemical and process addictions in the United States in terms of economics, health care, work productivity, family life, crime, social welfare, and mental health are high (Juhnke \& Hagedorn, 2006). It is estimated that alcohol and drugs cost the U.S. economy $\$ 365.5$ billion a year, and Americans spend $\$ 57$ billion on illicit drugs (Junke \& Hagedorn, 2006). Economic costs alone, without factoring in the enormous emotional costs, show that addiction is clearly a major public health issue in the United States.

\section{Eye Movement Desensitization and Reprocessing Addictions Treatment}

Although there are a few articles and chapters describing the use of EMDR in treating addictions, there is very limited research investigating this intervention. Shapiro, et al., (1994) were among the first authors to address how EMDR could be effective in treating substance abuse. They indicated that EMDR could be used to desensitize memories that contributed to substance use, triggers of use, and the relapse process. In targeting and reprocessing these memories, the authors indicated that the client would have a greater chance of engaging with recovery and maintaining sobriety.

Zweben and Yeary (2006) reported on the potential uses of EMDR in addictions treatment. They explored how trauma treatment using EMDR could be integrated into various levels of care. These authors noted that abstinence may be supported by helping clients stabilize their functioning by developing internal resources to self-soothe. However, "the affects that cause the desire for self-medication and relapse potential are inherent within the unprocessed memories and should be dealt with (via) the standard EMDR protocols" (p. 124).

Cox and Howard (2007) discussed the relationship between trauma and sex addiction. They integrated EMDR with other interventions to treat the shame and trauma associated with a client's sexual abuse that was at the root of his sexual addiction. Treatment was successful in relieving the client's distress associated with his abuse history and in preventing relapse.

Hase, Schallmayer, and Sack (2008) completed a randomized control study on EMDR and addiction.
Thirty-four patients with chronic alcohol dependence were randomly assigned to treatment as usual or treatment as usual plus two EMDR sessions, using a protocol focused on the addiction memory (AM; Hase, 2006). Findings indicated that patients receiving EMDR sessions had significantly less craving than the control group after terminating inpatient treatment and at 1-month follow-up. EMDR treatment was also associated with fewer relapses and a decrease in depressive symptoms.

Marich (2009) examined the treatment of a crossaddicted female (alcohol, cannabis, and sedatives) using EMDR. The author demonstrated how trauma treatment enhanced the client's progression through the 12-step model of recovery. Negative cognitions (and their related memories) were targeted to allow the client to free herself from blocking beliefs that might impede her recovery. The client had maintained sobriety and made other significant life changes at 18-month follow-up.

Abel and O'Brien (2010) reported on the use of EMDR in treating a female with comorbid PTSD and alcohol dependence. The authors explored the decisions regarding the use of the standard EMDR protocol for trauma previous to the client achieving sobriety. These authors argued that targeting underlying trauma using the standard EMDR protocol is necessary for certain clients to be able to achieve and maintain sobriety.

\section{Addiction Treatment Protocols}

Clients with addiction issues often have great difficulty in maintaining sobriety/behavior change. Clinicians may use EMDR and pre-EMDR strategies in assisting clients to develop coping skills, increasing affect tolerance, and supporting sobriety. The following protocols are commonly used in treating clients who present with addictions: Desensitization of Triggers and Urge Reprocessing (DeTUR; Popky, 2005), CRAVEX (Hase, 2010), Resource Development Installation (Korn \& Leeds, 2002), and Affect Tolerance (York \& Leeds, 2001).

Desensitization of Triggers and Urge Reprocessing. The Desensitization of Triggers and Urge Reprocessing (DeTUR) protocol (Popky, 2005) was developed to help clients reinforce positive coping by focusing on both treatment goals and relapse triggers. In the first part of the DeTUR protocol, the client focuses on what is called the positive treatment goal. Each client develops an image of what life would look like when changes are made in alcohol and/or drug consumption, whether the change is abstinence or harm 
reduction. After enhancing this goal through the use of visual imagery reinforced by bilateral stimulation, the clinician works with the client to develop both internal and external resources to support change. The clinician and client then work to desensitize each trigger for the client's use of substances. Clients are taught to use bilateral stimulation on their own if urges recur.

Michael Hase's Memory of Addiction/CRAVEX Protocol. The Hase Memory of Addiction/CRAVEX Protocol (Hase, 2010) specifically targets the memory of a client's last craving/urge or relapse using the broad general outline of the standard EMDR protocol. Reprocessing of the addiction memory (AM) leads to both a decrease in cravings or urges and potentially to channels in the brain connected to the reasons why the individual initially became addicted. Instead of a SUDS (Subjective Units of Distress) rating, the protocol calls for a rating of the level of urge (LOU) on a scale of 0 to 10 , where 0 is no urge and 10 is the highest urge one can imagine. The positive and negative cognitions are identified, as well as the emotions and the location of body awareness. Bilateral stimulation is used and the LOU is rated. After desensitizing the memory ( $\mathrm{LOU}=0)$, the client is assisted in installing the positive cognition.

Affect Tolerance Protocol. Poorly managed affect can be a primary trigger for relapse (Cummings, Gordon, \& Marlatt, 1980). The Affect Tolerance Protocol can be extremely effective when teaching clients to tolerate feelings (York \& Leeds, 2001). The client is asked to identify a feeling that is creating distress and the worst part of this feeling. The client then develops an image of the feeling, a negative cognition, a positive cognition, validity of cognition (VOC), feelings about the feelings, SUDS, and body sensations. The goal of this protocol is to lower the SUDS, not to get to zero. After desensitizing with bilateral stimulation, the clinician returns to the target image and checks the SUDS. If the SUDS does not go down after several sets, the clinician prompts the client to use other affect management techniques, such as breathing and talking, to lower the emotional arousal. The positive cognition is then installed. There is no body scan used at the end of the session.

Resource Development Installation Protocol. Clients with addictions often need to enhance their relapse prevention plan by strengthening qualities or resources for coping. These clients may benefit from the use of the Resource Development Protocol (Korn \& Leeds, 2002). After identifying a challenging current life situation where increased coping is needed, the client develops an image of this situation and rates the SUDS. The clinician directs the client to identify a personal life experience in which he or she exhibited the resource, a figure who embodies the resource, or an image that represents it. Each of these exemplars of the resource is then enhanced by identifying an image that represents it, as well as sounds and body sensations. Bilateral stimulation is used to reinforce these representations. The client then returns to the image of the challenging life situation and imagines how he or she will use the newly developed qualities to enhance coping.

\section{The Stages of Change}

Prochaska and DiClemente $(1982,1992)$ developed a model that described the stages through which people progress as they engage in behavior change. These authors identified the following stages of change: precontemplation, contemplation, preparation, action, maintenance, and relapse and recycling. Research has validated the existence of these various stages in clients presenting to addictions treatment facilities or in community samples (Demmel, Beck, Richter, \& Reker, 2004; Hartney et al., 2003), as well as the success of this model in adapting treatments for addictions and compulsive behavior (Armitage, 2006; Berman, Forsberg, Durbeej, Kallman, \& Hermansson, 2010; DiClemente, Schlundt, \& Gemmell, 2004; Giovazolias \& Davis, 2005; Mitchell \& Angelone, 2006; Wade, Frayne, Edwards, Robertson, \& Gilchrist, 2009).

\section{The Road Map Model for Addiction Treatment}

Addictions are well established as a problem in the American population, yet clients who have a problem with substance and/or process addictions may be in various stages of awareness of their issues. Although a growing body of research indicates that EMDR can be used as an effective treatment for clients with addictions and several protocols that target the addiction directly are available, a significant gap exists in the literature about how to integrate EMDR into treatment with clients who are in various stages of change in respect to their addictions. In this article, the authors attempt to address the gap by providing a road map in using EMDR with clients in each of the stages of change.

The proposed model incorporates the following key elements:

1. Addiction treatment must be individualized; not all treatments work for all clients (NIDA, 2009).

2. Clients differ in terms of motivation for change. Clinicians need to be mindful of client readiness to change in designing effective treatment (Connors, Donovan, \& DiClemente, 2001). 
3. Trauma underlies addiction for many individuals (Peirce, Kindbom, Waesche, Yuscavage, \& Brooner, 2008).

4. Incorporating EMDR into addictions treatment involves complex decisions and must be done judiciously (Shapiro, Vogelmann-Sine \& Sine, 1994).

The authors offer this road map to help clinicians make clinical decisions about when to incorporate EMDR into addictions treatment. What follows is an expanded discussion of the stages of change and specific interventions which may be used at each stage. For the purposes of this article, the authors use the terms "EMDR" and "pre-EMDR" to distinguish between techniques. Pre-EMDR involves those interventions which prepare a client for adaptive information processing in the future and help to develop a client's ego strengths, resources, and affect tolerance. We use the term EMDR to refer to trauma processing in Phases 4-7.

\section{Precontemplation Stage}

Although on some level, the client may recognize that there is a problem, individuals in this stage have no intention of changing behaviors in the next 6 months. The client sees his or her addiction as far more positive than negative. Clients may present as being in denial, minimizing, rationalizing, and/or resistant to change.

The goal with this group of clients is to help them to begin to think about making a change. Interventions at this stage include inviting the client to engage in a process of contemplating the issue. Education about the effects of addiction can be helpful. Clients may benefit from consciousness raising. For example, one may help the client make the previous unconscious connection between his or her addiction and certain behaviors or problems. Change is painful, and the negative consequences of addiction must outweigh the pain of change for clients to be motivated to make these difficult lifestyle changes (Shaffer \& Jones, 1989).

EMDR Protocols. In this stage, EMDR interventions are somewhat limited in scope. Interventions may include the use of the Affect Tolerance Protocol as a way to help clients begin to learn to manage feelings. The Resource Development Protocol may also be used to help the client cope with other issues (i.e., anxiety, daily life stresses).

\section{Contemplation Stage}

Individuals who are in the contemplation stage have begun considering ways that they wish to change their behavior regarding addiction, but they have not yet taken any steps to do so. Plans may include the intention to reduce use or quit the addiction completely. Often, people in this stage are experiencing some distress and discomfort as a result of their use and may be considering the benefits of a lifestyle change (Prochaska \& DiClemente, 1982, 1992).

These clients in the contemplation stage are thinking about changing. They are not yet ready to actually make a change, but they are at the point of considering it. They have probably begun to experience some pain around their use, such as negative feedback from others, physical problems, and embarrassment about behaviors. Essentially, clients in this stage are doing a cost-benefit analysis. Change is extremely difficult for people and the pain of change often outweighs the pain of staying the same. These people are able to make some connection between their addiction and some of the pain they experience, but they are often highly ambivalent about giving up something that has served them well in the past (Shaffer, 1994).

People who use drugs and alcohol or who engage in compulsive behaviors do so because it is a mostly positive experience, particularly in the beginning. People experience the benefits of using (e.g., a sense of peace, relaxation, social disinhibition, energy changes). As time passes, negative experiences start to occur and begin to accumulate. It is only then that the person begins to question these behaviors. Shaffer and Jones (1989) call these moments when the pain outweighs the pleasure of using "turning points." The moments add up to an experience of difficulty rather than pleasure. Often, there is one major turning point when the person finally makes a choice to change. In the addiction field, this is also known as "hitting bottom." After the individual realizes that he or she has a problem and wants to quit, it can then be days, weeks, or years before the person actually makes a change. In some cases, the person may never make the change.

Therapists can intervene in this stage by helping clients to become more aware of choices and decide about whether and when to make a change.

Pre-EMDR Protocols. The following interventions may be helpful at this stage:

Robin Shapiro's Two-Handed Interweave (Shapiro, 2006). This is a very useful technique that was developed by Robin Shapiro to help a client who is ambivalent or having difficulty making a decision. The client places the idea of one feeling, thought, or belief in one hand and the opposite feeling, thought, or belief in the other hand. Bilateral stimulation is used while the client notices what is going on in each hand. The client may also alternate opening and closing each hand. 
After this process, the client is encouraged to discuss what is learned (Shapiro, 2006).

Resource Development Installation (RDI; Korn \& Leeds, 2002). Clients who are ambivalent about change may have some barriers to being more committed to the change process. They may worry about the following: How will I manage social functions without alcohol? How can I negotiate conflict with my partner without drinking? What will I do when my gambling friends want me to go with them to the racetrack? RDI can be used to enhance qualities that a client may need to develop before preparing to make the actual change.

DeTUR Positive Treatment Goal. The DeTUR positive treatment goal can be a useful intervention for clients in this stage. Clients who are ambivalent about change may need assistance in reinforcing what the benefits of that change could be. The DeTUR positive treatment goal may be used to create the ideal vision of what the client's life would look like if he or she made the change. Bilateral stimulation can be used to reinforce these images.

\section{Preparation Stage}

At this point, the client has made a commitment to change. He or she develops goals, priorities, and a practical change plan. The client may also experience some ambivalence and go back and forth about what action to take. Individuals do not automatically move into the next stage of action from this stage. It may take weeks, months, or even years for that to happen. The actual change may never occur, even though the client has entered the preparation phase.

In this stage, therapists help clients to develop their change plans. This could include helping clients to explore what lifestyle changes are necessary to support the change and exploring possible treatment programs, such as inpatient and intensive outpatient programs. Clients who are physically dependent on substances should be encouraged to go to inpatient detoxification programs. Self-help groups may be explored.

Medications. Medications may be used to support change. There are several approved treatments for drug and alcohol dependence (Kampman, 2009). These FDA-approved medications include those for alcohol (disulfiram, naltrexone, acamprosate), opiates (methadone, buprenorphine, Suboxone, naltrexone), and nicotine (nicotine replacement, bupropion, varenicline). In addition, clients with co-occurring depression, anxiety, and other psychiatric illness may need to consider how psychiatric medications can be an important support to recovery.
Pre-EMDR and EMDR Protocols. At this point, EMDR treatment interventions that would be appropriate include using the DeTUR Protocol's positive treatment goal to reinforce the client's decision to develop a changed lifestyle. Clients may benefit from the use of the RDI Protocol to reinforce the qualities necessary to maintain the changes. In addition, the Affect Tolerance Protocol may be used to help clients manage any difficult feelings that emerge. Considering whether it is appropriate to use the standard EMDR protocol for traumas at this point is a serious clinical consideration, which will be discussed subsequently.

\section{Action Stage}

Clients in the action stage are ready to make a clear decision and commitment to change and develop behavior consistent with this new lifestyle (Connors, Donovan \& DiClemente, 2001). Clients initiate the actual change and begin to make necessary adjustments to support new behaviors. Change is accomplished by implementing the plans that were made during the preparation phase, such as maintaining a drug-free and alcohol-free environment, changing the way the client socializes, developing new coping mechanisms and skills, and modifying problem behaviors. Some people may choose not to quit their addiction completely but may develop strategies to cut back. Therapists working with action stage clients can assist by encouraging new behavior and helping them manage urges and triggers. Clients may benefit from learning healthy ways to deal with stress, setting up exercise plans, discovering new ways to have fun and relax, as well as identifying and managing triggers for relapse. This is a short stage that lasts up to 6 months.

\section{Pre-EMDR}

Affect Tolerance Protocol. The Affect Tolerance Protocol can be used to help clients manage the overwhelming emotions that may emerge during the action stage. Cummings et al. (1980) identified the three primary events that accounted for $70 \%$ of relapses for those in recovery from chemical and behavioral addictions (negative emotional states, social pressure, interpersonal conflict). Negative emotional states were named as the highest trigger of old behavior, accounting for $35 \%$ of relapse experiences. Clients need to be educated about how negative affect could jeopardize their recovery.

Resource Development Installation. Clients in the action phase may find that they need to create additional resources to handle the stresses of the change process. 
RDI (Korn \& Leeds, 2002) can be implemented to create these new strategies. Bilateral stimulation can be used to integrate a future template of the client managing these challenges with the addition of the new resource.

\section{EMDR Protocols}

Hase/CRAVEX Protocol. As clients implement their action plan for recovery, they may experience urges to return to old behaviors. The therapist may use the Hase/CRAVEX protocol with clients to desensitize these episodes and to determine the factors that lead to the urge. Therapy can then target these issues by reinforcing previous coping skills or developing new skills as needed.

DeTUR Protocol. The DeTUR protocol is another useful tool to help clients manage urges and to prevent relapses. The protocol may be used to help clients identify triggers and desensitize them.

Standard EMDR Protocol. The decision about when and whether to specifically target a client's trauma is complex. Is the client stable enough to deal with processing trauma? Does he or she need to target his or her traumatic memories to maintain change? Is the trauma contributing to the addictive process or is it only peripherally related? Treatment must explore these complicated situations. If the trauma is to be targeted, the standard EMDR protocol can be used to disconnect the trauma-addiction link. This will be discussed subsequently.

\section{Maintenance Stage}

Maintenance is the long-term continuation of the changes that have been implemented during the action phase. Permanent lifestyle changes must be implemented. Triggers, the events, or stimuli in the environment and/or within the person that create cravings and desires to return to old behaviors must be explored, and alternative strategies should be developed. Long-term conflicts should be resolved. Shaffer and Jones (1989) studied cocaine addicts who recovered without any outside intervention. These individuals reported that four things helped maintain recovery: new friends and relationships with nonusers, physical exercise, healthy eating habits, and a spiritual belief system. The focus in this stage of maintenance is relapse prevention.

Pre-EMDR and EMDR Protocols. In the maintenance stage, there are many opportunities to intervene with EMDR. The Hase/CRAVEX Protocol may be used to desensitize urges and cravings. The DeTUR protocol may also be used to identify and desensitize triggers. It may be very helpful to use the Affect Tolerance Protocol to help clients manage many difficult feelings, which emerge in this stage of recovery. The RDI protocol can be used to shore up inner resources and build ego strength. Once a period of sobriety or abstinence has been established and ego strength is developed, many clients become more ready and able to do trauma work to resolve underlying issues. At this point, many can benefit from working with the standard trauma protocol.

\section{Relapse and Recycling Stage}

If a client relapses and old behaviors recur, he or she leaves the action or maintenance stage. He or she must then reevaluate motivation for change from this new point of view. Some may return to precontemplation or contemplation, whereas others may be further along in the process and immediately jump back into preparation or action.

The clinician helps the client distinguish whether this is a one-time lapse or a more serious relapse, that is, a return to the former patterns of behavior (Marlatt \& Gordon, 1985). The therapist may help the client see the lapse or relapse as part of a learning process; the client has the potential to learn something necessary and important about the reasons for relapse as well as what is needed to resume the recovery process. The triggers leading to the relapse are analyzed.

Therapists help clients with this evaluation of motivation for change to determine which stage the client is now in and then intervene appropriately.

Pre-EMDR and EMDR Protocols. Once the therapist determines the client's motivation to change, the interventions discussed previously for each stage may be appropriate. EMDR interventions, which may be useful in this stage, include the Hase/CRAVEX Protocol to specifically target the relapse event. As in other stages, using the Affect Tolerance Protocol, RDI, DeTUR, and the standard EMDR protocol may be helpful.

\section{When to Use the Standard EMDR Protocol for Clients With Traumatic Memories}

How does one decide to do trauma work when addiction is present? This is an important clinical decision and must be considered carefully. There is some controversy in the EMDR literature as to when the use of this protocol is appropriate. Some believe the client should be sober first (Hase, 2010), whereas others (Abel \& O’Brien, 2010; Shapiro, Vogelmann-Sine, \& Sine, 1994) 
believe that the client may not be able to maintain sobriety or abstinence until the traumas are resolved.

Abel and O'Brien (2010) outlined various factors to consider when making a decision about using the standard EMDR protocol for trauma processing. The following are an expansion of those ideas:

\section{What is the client's stage of change? What is the client motivation?}

This is probably the key factor. If the client is not ambivalent, is in the preparation or action phase, and is seriously motivated to do the work, the outcome may be more positive.

2. The person's addiction history: Did their trauma trigger their use? Shapiro et al. (1994) noted that many addicts have a trauma history of early abuse, neglect, or poor attachment. If the trauma preceded the addiction, the trauma may need to be resolved for the client to maintain abstinence.

3. The client's history of trying to quit: What happens when a client tried to quit?

How many times did this occur? What was the client's experience? Some clients will report that they relapsed after trying to quit because they were experiencing memories of abuse or trauma. Explore this.

4. What internal/external resources does the person have?

To be successful using the trauma protocol, clients must have some sense of internal and external support even if they are continuing to use substances. They will need these resources to be successful with the trauma processing (Korn $\&$ Leeds, 2002).

5. Does the client have a single or complex trauma history?

Obviously, complex trauma is much more difficult to deal with and will involve a longer treatment process. Sobriety or abstinence may be more important with these individuals before embarking on the trauma work, as they need longer periods of stabilization before proceeding. If the trauma is a single or a simple trauma and if it is linked in any way to the addiction, then doing the trauma work first may be helpful.

6. What is the client's functional level?

Is the client employed and able to function independently in his or her life? Consider a client's functional level and whether or not trauma work may disrupt his or her ability to continue at this level.

7. What is the client's current stress level? Stress is a natural part of life. However, if the client is currently experiencing significant stressors aside from their trauma symptoms (e.g., job loss, severe financial problems), he or she may already be close to his or her limit of tolerating stress. Trauma-focused work will likely need to wait until the client is more stabilized.

8. Does the client have affect tolerance?

The client must have some ability to tolerate affect to process the trauma (York \& Leeds, 2001). If not, the processing may be too destabilizing and should wait until there is some recovery from the addiction.

9. Does the client need detoxification?

If the client is physically dependent on the chemical, inpatient or a monitored outpatient detoxification should probably happen prior to the trauma processing.

10. What is the quality of the therapeutic relationship?

We believe that the therapeutic relationship is a critical factor to consider when determining whether to proceed with the trauma protocol. Much of the healing occurs in therapy through the positive use of the therapeutic relationship (Najavits et al., 1995). If the therapist can provide a safe and protected place for the client, the client may be more willing to proceed with what is potentially a difficult piece of clinical work. In addition, the therapist must also be committed to the relationship and to being available to the client through times of distress.

\section{Discussion}

The treatment of addiction is complex because each person is a unique individual who deserves a personal assessment and an individual treatment plan. There is no one "cookie cutter" approach that will work for all clients. However, addiction treatment programs often find it difficult to individualize care, despite knowledge that this will provide the best outcomes (Mericle, Casaletto, Knoblach, Brooks, \& Carise, 2010).

An accurate assessment of addiction involves looking at a client's motivation to change. Prochaska and DiClemente $(1982,1992)$ provide a very helpful model for assessing motivation. Their stages of change have been discussed in this article, as well as appropriate pre-EMDR and EMDR interventions. Treatment must be tailored to fit the stage in which the client presents. Accurate treatment matching allows for a better chance of engaging with the client, a better chance of meeting the clients' needs, and an increased potential for change.

\section{EMDR and the Stages of Change}

EMDR is a very efficient addition to a clinician's tool bag of interventions when dealing with addiction. 
The pre-EMDR and EMDR interventions described in this article provide helpful supports to a client's potential recovery, particularly if a client has had prior treatment which has not been successful. The authors have considerable experience working with addictions and have found that EMDR, coupled with more traditional substance abuse interventions, provides a very effective component of treatment. In the following section, we look at how each of the EMDR interventions can be used in the various stages of change.

The safe place exercise is a useful place to start with any client and can be used even in the precontemplation stage of change. This exercise helps clients to learn to relax and to manage stress. Clients can use this as a foundation exercise to return to repeatedly during treatment. It also allows the clinician to see how open and ready a client might be for the use of bilateral stimulation.

RDI provides many benefits as well. It helps shore up an individual's ego strengths for what could be a rocky road of recovery. RDI strengthens a client's foundational coping skills and allows them to prepare for future work. It can be used effectively in all the stages of change.

The Affect Tolerance Protocol helps clients manage the difficult feelings that may emerge in recovery. Clients in addiction treatment often need to enhance their coping to deal effectively with the underlying feelings that led to use in the first place. By rehearsing effective affect management in session, the client can avoid relapses caused by negative affect states.

The Hase/CRAVEX protocol is a particularly efficient way to manage cravings, urges, and recent relapse. The protocol can assist the client by desensitizing urges and cravings and may also help the client to explore the underlying causes for addiction. It is useful because it is used to target the last urge or relapse while it is fresh in a client's mind.

The initial component of the DeTUR protocol incorporates the use of the positive treatment goal that enhances client recovery by providing an image of how much better life could be without addiction. The remainder of the protocol is a bit more difficult for clients to use and appears less efficient because desensitizing the urges and triggers for use is often a longer process. A client needs to commit to desensitizing all the triggers; this includes breaking down each trigger into steps. For example, if a client is triggered by coming home from work, all of the minor triggers involved, such as getting into the car, driving home, and getting off the exit also need to be targeted. This is a longer and more arduous process than the Hase/CRAVEX Protocol.
The issue of if and when to use the standard EMDR protocol for trauma with individuals who have addictions or who abuse substances is a complex decision. The authors believe it is a very important part of recovery for many clients. Although it should be used judiciously, there are some clients who will not or cannot enter into recovery until the underlying trauma issues are resolved.

\section{Recommendations for Future Research}

The research literature continues to examine ways to effectively treat clients who present with various levels of chemical and process addictions. Current models are heavily focused on examining the ways that addiction changes brain structure and how to incorporate this awareness into treatment (van Wormer $\&$ Davis, 2008). EMDR fits perfectly with this focus on biological processes and can be an important part of addictions treatment and research designs.

More research is needed to clarify ways by which EMDR can be used as a primary or adjunctive treatment for clients with addictions and to further explore the applications of the road map model for addiction treatment. Some of the questions that research might explore include the following:

1. Does a client have to be sober to benefit from the standard EMDR protocol? If so, how long must the client be sober? If not, under what circumstances would it be advisable for a clinician to proceed?

2. How does the use of various EMDR protocols augment addiction treatment? Do specific protocols work more effectively with certain clients? Certain addictions?

3. How can EMDR treatment most effectively target specific relapse triggers?

Research might focus on clients presenting for addictions treatment while in different levels of care. Various pre-EMDR and EMDR interventions could be implemented to examine what works best with clients in various stages of change and in various treatment settings. Research may follow up on Hase et al. (2008) to compare addiction treatment as usual to addiction treatment with EMDR. Various protocols (e.g., Hase/Cravex, DeTUR) could also be compared and contrasted.

\section{Summary}

The authors have presented a model for treating addictions using EMDR and non-EMDR techniques based on client readiness to change. The stages of change developed by Prochaska and DiClemente $(1992,1998)$ 
were described. Pre-EMDR and EMDR interventions were suggested for each stage. The process of treatment planning is conceptualized in terms of developing a road map. There are many turns in any road and many choices about which way to travel; clients and clinicians need to be aware of these choices as they travel together down the path to recovery.

\section{References}

Abel, N. J., \& O’Brien, J. M. (2010). EMDR treatment of comorbid PTSD and alcohol dependence: A case example. Journal of EMDR Practice and Research, 4, 50-59.

American Psychiatric Association. (2004). Practice guidelines for the treatment of patients with acute stress disorder and posttraumatic stress disorder. Arlington, VA: Author.

Armitage, C. J. (2006). Evidence that implementation intentions promote transitions between stages of change. Journal of Consulting and Clinical Psychology, 74(1), 141-151.

Berman, A. H., Forsberg, L., Durbeej, N., Källmén, H., \& Hermansson, U. (2010). Single-session motivational interviewing for drug detoxification inpatients: Effects on self-efficacy, stages of change and substance use. Substance Use \& Misuse, 45(3), 384-402.

Buchman, D., \& Reiner, P. B. (2009). Stigma and addiction: Being and becoming. The American Journal of Bioethics, 9(9), 18-19.

Connors, G. J., Donovan, D. M., \& DiClemente, C. C. (2001). Substance abuse treatment and the stages of change. New York: Guilford Press.

Cox, R. P., \& Howard, M. D. (2007). Utilization of EMDR in the treatment of sexual addiction: A case study. Sexual Addiction and Compulsivity, 14(1), 1-20.

Cummings, C., Gordon, J. R., \& Marlatt, G. A. (1980). Relapse: Prevention and prediction. In W. R. Miller (Ed.), The addictive behaviors (pp. 291-321). New York: Pergamon.

Del Giudice, M. J., \& Kutinsky, J. (2007). Applying motivational interviewing to the treatment of sexual compulsivity and addiction. Sexual Addiction and Compulsivity, 14(4), 303-319.

Demmel, R., Beck, B., Richter, D., \& Reker, T. (2004). Readiness to change in a clinical sample of problem drinkers: Relation to alcohol use, self-efficacy, and treatment outcome. European Addiction Research, 10(3), 133-138.

DiClemente, C. C., \& Prochaska, J. O. (1998). Toward a comprehensive, transtheoretical model of change: Stages of change and addictive behaviors. In W. R. Miller \& N. Heather (Eds.), Treating addictive behaviors (2nd ed., pp. 3-24). New York: Plenum Press.

DiClemente, C. C., Schlundt, D., \& Gemmell, L. (2004). Readiness and stages of change in addiction treatment. The American Journal on Addictions, 13(2), 103-119.

Dittmar, H. (2005). Compulsive buying-a growing concern? An examination of gender, age, and endorsement of materialistic values as predictors. British Journal of Psychology, 96(Pt. 4), 467-491.
Drake, R. E., \& Mueser, K. T. (1996). Alcohol-use disorders and severe mental illness. Alcohol Health and Research World, 20(2), 87-93.

Foa, E. B., Keane, T. M., Friedman, M. J., \& Cohen, J. A. (2009). Effective treatments for PTSD: Practice guidelines from the International Society for Traumatic Stress Studies (2nd ed.). New York: Guilford Press.

Giovazolias, T., \& Davis, P. (2005). Matching therapeutic interventions to drug and alcohol abusers' stage of motivation: The clients' perspective. Counselling Psychology Quarterly, 18(3), 171-182.

Hartney, E., Orford, J., Dalton, S., Ferrins-Brown, M., Kerr, C., \& Maslin, J. (2003). Untreated heaving drinkers: A qualitative and quantitative study of dependence and readiness to change. Addiction Research and Theory, 11(5), 317-337.

Hase, M. (2006, September). Reprocessing of the addiction memory. Paper presented at the Annual EMDR International Conference, Philadelphia, PA.

Hase, M. (2010). CraveEx: An EMDR approach to treat substance abuse and addiction (pp. 467-488). In M. Luber (Ed.), EMDR scripted protocols. New York: Norton.

Hase, M., Schallmayer, S., \& Sack, M. (2008). EMDR reprocessing of the addiction memory: Pretreatment, posttreatment and 1-month follow-up. Journal of EMDR Practice and Research, 2(3), 170-179.

Hollander, E., \& Allen, A. (2006). Is compulsive buying really a disorder and is it really compulsive? American Journal of Psychiatry, 163(10), 1670-1672.

Ironson, G., Freund, B., Strauss, J. L., \& Williams, J. (2002). Comparison of two treatments for traumatic stress: A community-based study of EMDR and prolonged exposure. Journal of Clinical Psychology, 58(1), 113-128.

Jacobsen, L. K., Southwick, S. M., \& Kosten, T. R. (2001). Substance use disorders in patients with posttraumatic stress disorder: A review of the literature. The American Journal of Psychiatry, 158(8), 1184-1190.

Juhnke, G. A., \& Hagedorn, W. B. (2006). Counseling addicted families: An integrated assessment and treatment model. New York: Taylor \& Francis.

Kampman, K. M. (2009). Biologic treatments for drug and alcohol dependence. Primary Psychiatry, 16(8), 47-52.

Korn, D. L., \& Leeds, A. M. (2002). Preliminary evidence of efficacy for EMDR resource development and installation in the stabilization phase of treatment and complex posttraumatic stress disorder. Journal of Clinical Psychology, 58(12), 1465-1487.

Lee, C. W., Taylor, G., \& Drummond, P. D. (2006). The active ingredient in EMDR: Is it traditional exposure or dual focus of attention? Clinical Psychology and Psychotherapy, 13, 97-107.

Marich, J. (2009). EMDR in the addiction continuing care process: Case study of a cross-addicted female's treatment and recovery. Journal of EMDR Practice and Research, 3(2), 98-106.

Marlatt, G. A., \& Gordon, J. R. (1985). Relapse prevention: Maintenance strategies in the treatment of addictive behaviors. New York: Guilford Press. 
Mericle, A., Casaletto, K., Knoblach, D., Brooks, A., \& Carise, D. (2010). Barriers to implementing individualized substance abuse treatment: Qualitative findings from the CASPAR Replication studies. Journal of Drug Issues, 40(4), 819-839.

Mitchell, D., \& Angelone, D. J. (2006). Assessing the validity of the Stages of Change Readiness and Treatment Eagerness scale with treatment-seeking military service members. Military Medicine, 171(9), 900-904.

Najavits, L. M., Griffin, M. L., Luborsky, L., Frank, A., Weiss, R. D., Liese, B. S., et al. (1995). Therapists' emotional reactions to substance abusers: A new questionnaire and initial findings. Psychotherapy: Theory, Research, Practice, Training, 32(4), 669-677.

National Institute on Drug Abuse. (2009). Principles of drug addiction treatment: A research-based guide (2nd ed.).Washington, DC: U.S. Government Printing Office. Retrieved September 18, 2010, from http:// www.nida.nih.gov/podat/podatindex.html

Peirce, J. M., Kindbom, K. A., Waesche, M. C., Yuscavage, A. S., \& Brooner, R. K. (2008). Posttraumatic stress disorder, gender, and problem profiles in substance dependent patients. Substance Use \& Misuse, 43(5), 596-611.

Popky, A. J. (2005). DeTUR, an urge reduction protocol for addictions and dysfunctional behaviors (pp. 167-188). In R. Shapiro (Ed.), EMDR solutions: Pathways to healing. New York: Norton.

Prochaska, J. O., \& DiClemente, C. C. (1982). Transtheoretical therapy: Toward a more integrative model of change. Psychotherapy: Theory, Research and Practice, 19(3), 276-288.

Prochaska, J. O., \& DiClemente, C. C. (1992). Stages of change in the modification of problem behaviors. In $\mathrm{M}$. Hersen, R. M. Eisler, \& P. M. Miller (Eds.), Progress in behavior modification (Vol. 28, pp. 183-218). Sycamore, IL: Sycamore.
Shaffer, H. J. (1994). Denial, ambivalence and countertransference hate. In J. D. Levin \& R. Weiss (Eds.), Alcoholism: Dynamics and Treatment (pp. 421-437). Northdale, NJ: Jason Aronson.

Shaffer, H. J., \& Jones, S. B. (1989). Quitting cocaine: The struggle against impulse. Lexington, MA: Lexington Books.

Shapiro, F., Vogelmann-Sine, S., \& Sine, L. (1994). Eye movement desensitization and reprocessing: Treating trauma and substance abuse. Journal of Psychoactive Drugs, 26(4), 379-391.

Shapiro, R. (2006). The two-hand interweave. In R. Shapiro (Ed.), EMDR solutions: Pathways to healing (pp. 160-166). New York: Norton.

Tamam, L., Zengin, M., Karakus, G., \& Ozturk, Z. (2008). Impulse control disorders in an inpatient psychiatry unit of a university hospital. Klinik Psikofarmakoloji Bulteni, $18,153-161$.

van Wormer, K. V., \& Davis, D. R. (2008). Addiction treatment: A strengths perspective. Belmont, CA: Thomson Brooks/Cole.

Wade, T. D., Frayne, A., Edwards, S. A., Robertson, T., \& Gilchrist, P. (2009). Motivational change in an inpatient anorexia nervosa population and implications for treatment. The Australian and New Zealand Journal of Psychiatry, 43(3), 235-243.

York, C., \& Leeds, A. M. (2001, June 21). Gate theory: An accelerated information processing model for developing functional state change. Paper presented at the EMDRIA Conference, Austin, TX.

Zweben, J., \& Yeary, J. (2006). EMDR in the treatment of addiction. Journal of Chemical Dependency Treatment, 8(2), $115-127$.

Correspondence regarding this article should be directed to John M. O’Brien, 465 Congress St., Suite 700, Portland, ME 04101. E-mail: jobinport@aol.com 